\title{
Whole Virus Detection Using Paper Potentiometry and Aptamer
}

\author{
Subhashish Dolai and Massood Tabib-Azar * \\ University of Utah \\ * Correspondence: azar.m@utah.edu
}

\begin{abstract}
Paper-based sensors, microfluidic platforms and electronic devices have attracted attention in the past couple of decades because they are flexible, can be recycled easily, environmentally friendly, and inexpensive. Here we report a paper aptamer-based potentiometric sensor to detect the whole Zika virus for the first time with a minimum sensitivity of $2.6 \mathrm{nV} / \mathrm{Zika}$ and the minimum detectable signal (MDS) of $1.2 \times 10^{6} \mathrm{Zika}$. Our paper sensor works very similar to a P-N junction where a junction is formed between two different wet regions with different electrochemical potentials near each other on the paper. These two regions with slightly different ionic contents, ionic species and concentrations, produce a potential difference given by the Nernst equation. Our paper sensor consisted of a 2-3 $\mathrm{mm}$ x $10 \mathrm{~mm}$ segments of a paper with a conducting silver paint contact patches on its two ends. The paper is soaked in a buffer solution containing aptamers designed to bind to the capsid proteins on Zika. Atomic force microscopy studies were carried out to show both the aptamer and Zika become immobilized in the paper. We then added the Zika (in its own buffer) to the region close to one of the silver-paint contacts. The Zika virus (40 $\mathrm{nm}$ diameter with $43 \mathrm{kDa}$ or $7.1 \times 10^{-20} \mathrm{gm}$ weight), became immobilized in the paper's pores and bonded with the resident aptamers creating a concentration gradient. The potential measured between the two silver paint contacts reproducibly became more negative as upon adding the Zika. We also showed that an LCD powered by the sensor, can be used to detect the sensor output.
\end{abstract}

Keywords: paper based sensor; whole virus; Zika; aptamer

\section{Introduction}

Sensors built with paper as substrate have interesting advantages. They usually have short response time, they are low-cost [1] and are flexible [2], [3]. They are also attractive, since they are biodegradable and suitable for mass deployment in resource limited areas and can be easily used by unskilled operators. Paper is also great medium for immobilizing and trapping and even in some cases binding with biomolecules. Its porous structure with large connected pores composed of cellulose fibers, allows to transport liquid by means of capillary forces and, hence, result in short response time. The porous structure also allows any particle or species to stay immobilized with the paper structure. Paper can also be functionalized with certain material for selective sensing like nitrocellulose paper used for immobilizing nucleic acid. Paper based potentiometric sensors have been reported for detecting many ions and proteins [4]-[7]. Potentiometric paper based sensors [1], [8]-[16] use the gradient of ion distribution on the paper to generate an open circuit voltage (Voc). There are many Zika sensors and detection methods reported in the literature. These include serum analysis using the antibody detection assays [17], [18] detection of viral RNA using molecular-based techniques like conventional polymerase chain reaction (PCR) and real-time reverse transcription polymerase chain reaction (RT-PCR) [19]-[25]. The techniques overall largely involve using viral antibody or DNA/RNA extraction and then labelled detection using fluorescent probes, providing high sensitivity and specificity. Most reported techniques require specialized equipment and expensive procedures and are time consuming. The paper-based sensor is fast, inexpensive and can be used in regions with limited resources. We show that a simple LCD can be used to measure the 
sensor output with the power generated with the sensor itself. The paper sensor reported here can be modified by replacing its aptamer with aptamers for other viruses, pathogens and even bacteria.

Our work shows for the first time that a potentiometric paper sensor can reliably detect a whole virus using standard A4 printer paper. Paper can be soaked with antigens or aptamers that become immobilized in the paper. The aptamers are single strand DNA structure that are artificially grown to detect biomolecules and pathogens with high specificity [26], [27]. When Zika was added to one side of the aptamer-soaked paper, it shifts the charge distribution in the paper and generates an electrochemical potential difference. Intimate electrical contacts with paper can be made with Graphene [15], [16] conducting glues and epoxies or silver paint used here. We also propose a concept device (Fig. 1(a)) which can be printed with contacts formed with silver paint or any conducting ink and connected to an LCD for electronic readout.

\section{Material and Methods:}

The experiment involved a sample holder with two electrodes fixed on a glass slide using copper tape and connected through a BNC cable to NI-USB 6341 DAQ with custom LabVIEW program. The program measures the open circuit voltage $\left(\mathrm{V}_{o c}\right)$ as a function of time. The glass slide inside a petri dish was covered with a grounded copper tape to eliminate static charges. The sensor paper strips $(0.2-0.3 \mathrm{~cm} \times 1 \mathrm{~cm})$ were manually cut from a standard printer A4 pages with silver paint (Ted-Pella) as contact electrode. The paper device was then presoaked in appropriate media like de-ionized (DI) water/buffer/aptamer and buffer/Zika introduced at the positive terminal. All paper sensors were from a single A4 sheet.

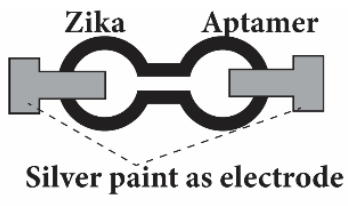

(a)

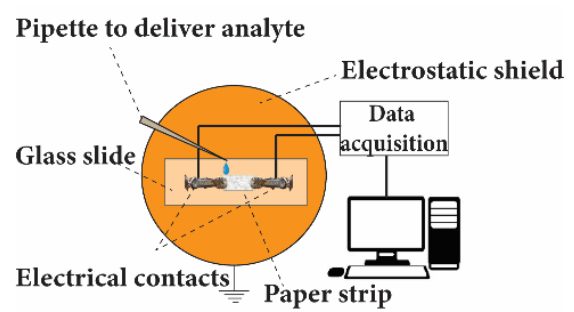

(b)

Figure 1. Schematic of the (a) proposed printed paper device. (b) experimental setup.

The buffer used was $1 x$ concentration of Phosphate Buffer Solution (PBS) with $1 \mathrm{mM}$ Magnesium Chloride. The aptamer has been prepared starting with mixing dried aptamer in resuspension buffer provided by BasePair Biotechnologies Inc. The aptamer solution obtained was then diluted to working concentration of $100 \mu \mathrm{M}$ using Aptamer Folding Buffer (BasePair product), with subsequently heating to $90^{\circ} \mathrm{C}-95^{\circ} \mathrm{C}$ for 5 minutes and successive cooling to room temperature for 15 minutes. The working aptamer obtained was then diluted to $1 \mu \mathrm{M}$ solution using buffer solution, prepared with 1x concentration of Phosphate Buffer Saline (PBS) and $1 \mathrm{mM}$ of Magnesium dichloride $\left(\mathrm{MgCl}_{2}\right)$. The $1 \mu \mathrm{M}$ aptamer had $\sim 1.2 \times 10^{12}$ number of aptamers in $2 \mu \mathrm{l}$ volume (calculated from the Avogadro number present in $1 \mathrm{M}$ concentration in 1 liter). The aptamer used in our experiment had a thiol end group of the 32 nucleotide-chain that folds to bind with Zika SF9 envelope protein [28]. Fig. 1A shows the schematic of predicted structure of the aptamer. The Zika was obtained from Zeptometrix and was diluted with $40 \mu \mathrm{l}$ of TCID_50 Zika stock solution in $60 \mu \mathrm{l}$ buffer. The number of Zika present in $2 \mu \mathrm{l}$ volume Zika solution was $\sim 7 \times 10^{6}$, estimated in same manner as in [30].

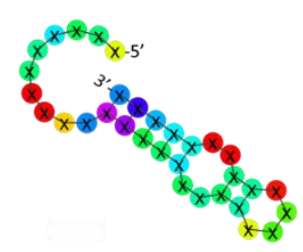


Figure 1A. Predicted structure of the aptamer (Image courtesy: BasePair Biotechnologies Inc. [29])

\section{Results and Discussions}

The open circuit voltage $\left(\mathrm{V}_{\text {oc }}\right)$ of a water-soaked paper device with buffer introduced on positive contact side is shown in Fig 2 as a function of buffer concentration. The $V_{o c}$ is given by the concentration gradient of the same ionic species $\mathrm{C} 1$ and $\mathrm{C} 2$ on both sides as in Nernst equation: $V_{o c}=$ $\frac{k T}{q} \ln \left(\frac{C 1}{c 2}\right)$, where $\frac{k T}{q}$ is the thermal voltage. The voltage reaches the highest value when the concentration gradient is at its peak. The $\mathrm{V}_{\text {oc }}$ also depends on the water content of the paper and paper material as well, which determines the diffusion rate of the ionic species introduced. The Zika virus becomes entangled in the pores of the paper and becomes immobilized. This results in a space charge region like a P-N junction diode giving rise to $\mathrm{V}_{\mathrm{oc}}$.

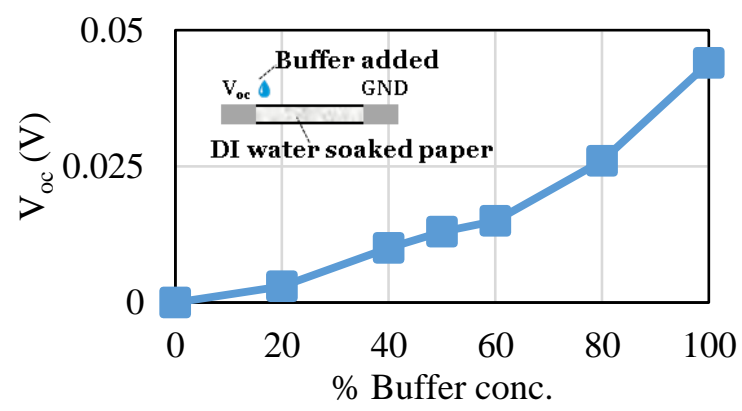

Figure 2. Voc vs percentage volume concentration of buffer with one side of the paper dipped in DI water and the other side with the diluted buffer solution.

The Zika virus has a small residual negative charge (shown in Fig 3(a)) that reduces the $\mathrm{V}_{\text {oc }}$ as soon as it was added to the paper strip. To demonstrate that the Zika (40 $\mathrm{nm}$ diameter) is immobilized on the paper, Zika stock solution was applied on a dry paper device. As the buffer spreads easily throughout the device, a depletion region between Zika+buffer and buffer maintain the $V_{\text {oc }}$ (Fig. 3). If Zika was also mobile, the resulting $V_{\text {oc }}$ will diminish as a function of time as the Zika diffuses.

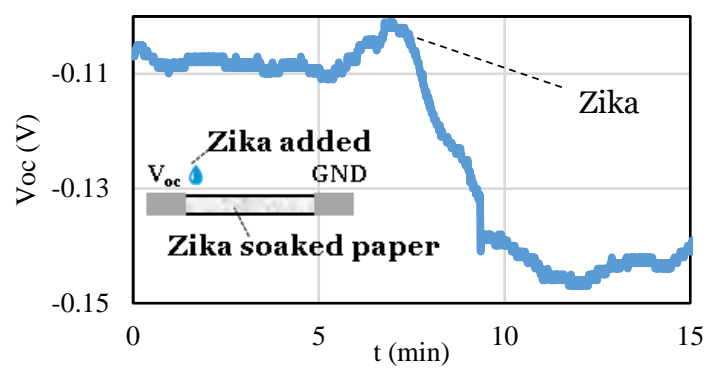

(a)

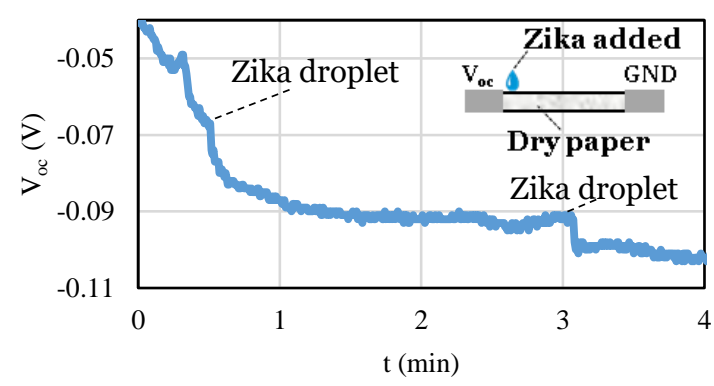

(b)

Figure 3. a) Zika introduced a) at $t=7.45 \mathrm{~min}$ to paper device presoaked in Zika to demonstrate net negative charge of the virus $b$ ) at $t=0.5 \mathrm{~min}$ and $\mathrm{t}=3.1 \mathrm{~min}$ to a dry paper device to demonstrate that as the Zika is applied on the positive side, it stays immobilized in the applied region as the buffer spreads maintaining the voltage level.

Fig 4 shows the $V_{\text {oc }}$ change $\left(\Delta \mathrm{V}_{\text {oc }}\right)$ with adding Zika on the positive side of the aptamer-soaked paper device as a function of Zika concentration. Zika while binding with aptamer causes charge redistribution of the aptamer-Zika. The successive addition of Zika showed reducing $V_{\text {oc. }}$ Once the aptamers were bonded with the Zika, the only charge rearrangement was caused by the Zika itself and not by the Zika-aptamer binding events. The average sensitivity had been measured to 2.6 $\mathrm{nV} / \mathrm{Zika}$ from the average $\Delta \mathrm{V}_{\text {oc }}$ of $\sim 18 \mathrm{mV}$ for $2 \mu \mathrm{l} \mathrm{Zika}$. The output response had a ripple noise voltage of $2 \mathrm{mV}$ and the minimum voltage it can detect is $1 \mathrm{mV}$, resulting in MDS of $1.2 \times 10^{6} \mathrm{Zika}$. 


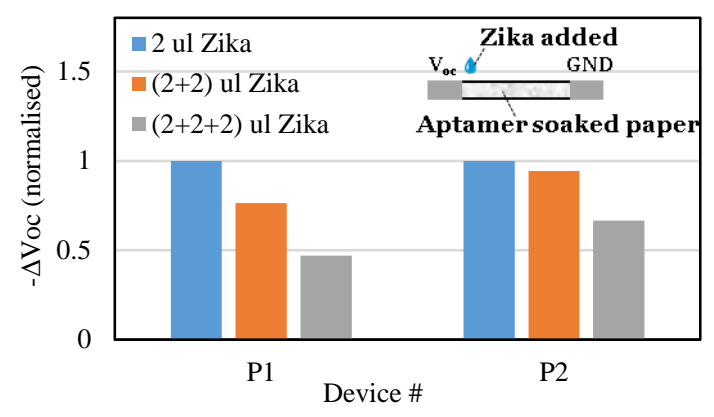

Figure 4. $2 \mu \mathrm{l}$ of diluted Zika added to aptamer-soaked paper at each instance (total 3 times) repeated on 2 separate paper devices (P1, P2).

The AFM scan of Zika in aptamer-coated paper (Fig 5(a)) shows the Zika virus. Due to high roughness of the paper structure a lower resolution scan was used. Another AFM scan of the Zika virus on aptamer-coated gold surface is shown in Fig 5(b) to clearly demonstrate the presence of the Zika virus on aptamer/gold sample. Our AFM studies on the stiction force of Zika in aptamer-coated paper shows higher stiction force with an AFM tip having an aptamer-coated bead (Fig 5(c)). The stiction force on aptamer-coated paper has been compared with respect to that of Zika/aptamercoated paper in Fig 5(d). The stiction force of Zika/aptamer is much higher due to aptamer specific binding with Zika.

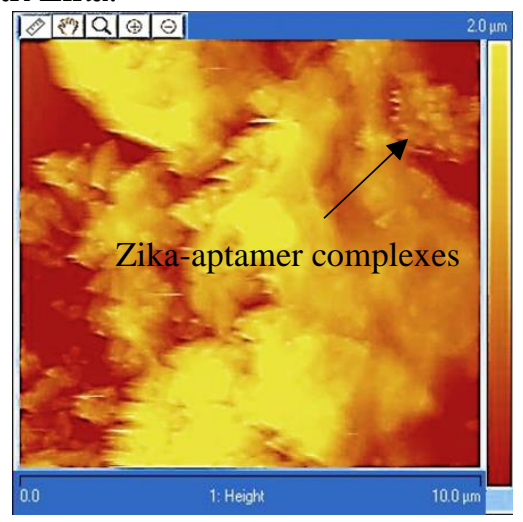

(a)

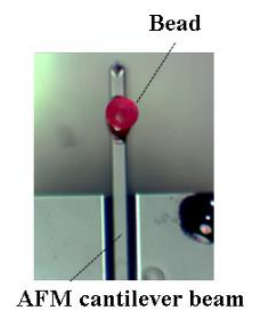

(c)

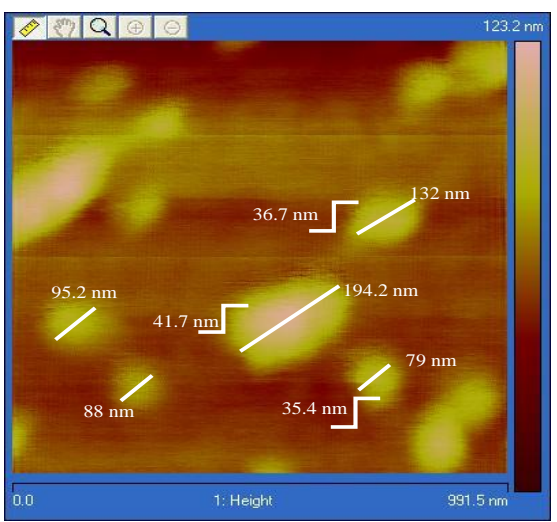

(b)

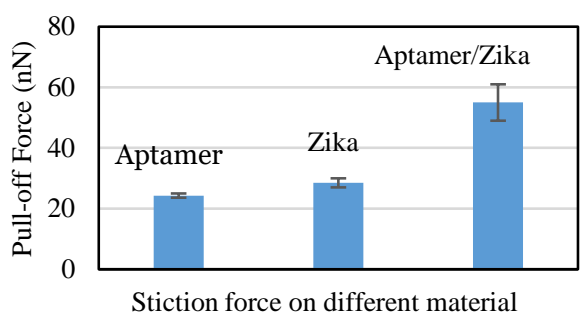

(d)

Figure 5. a) AFM image of the Zika on aptamer-coated paper. The scale is $10 \mu \mathrm{m}$. b) AFM image of Zika on gold substrate showing the Zika step height of $\sim 40 \mathrm{~nm}$ to demonstrate Zika on the aptamercoated gold surface. c) Photograph of the AFM tip with a single microbead (Diameter $150 \mu \mathrm{m}$ ). d) AFM stiction force measured using the AFM tip (c) with bead coated with aptamer specific to Zika. 


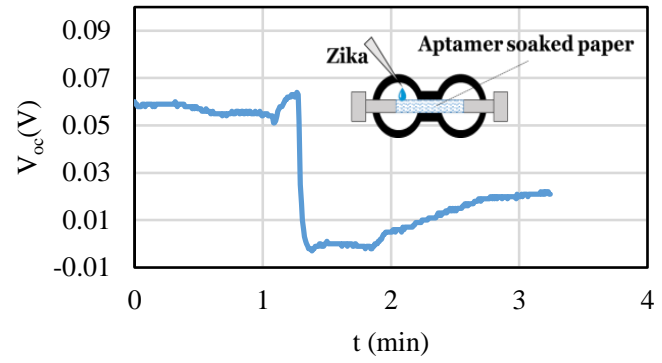

(a)

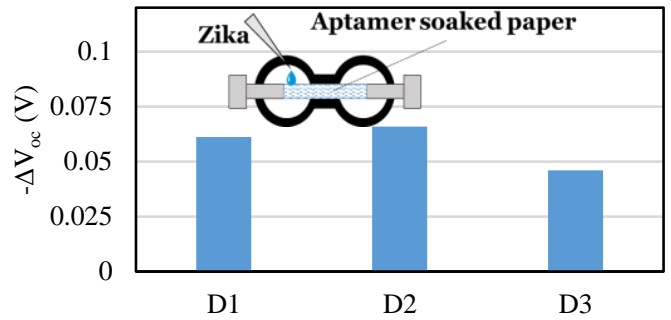

(b)

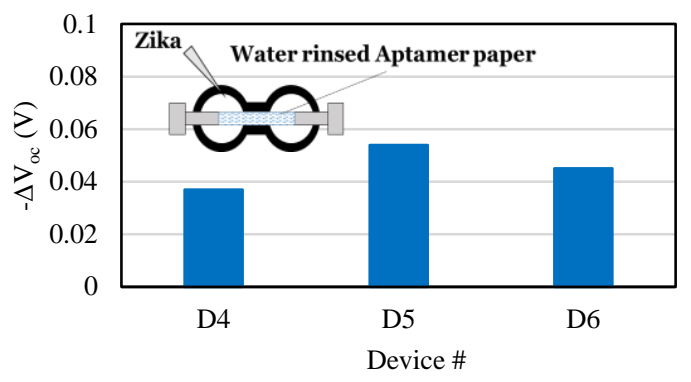

(c)

Figure 6. a) $\mathrm{V}_{\text {oc }} \mathrm{vs}$ time of the printed sensor with $\mathrm{Zika}$ added at $\mathrm{t}=1.3 \mathrm{~min}$. $\Delta \mathrm{V}_{\text {oc }}$ obtained on adding Zika to b) aptamer coated printed device D1, D2, D3, and c) aptamer coated and subsequently rinsed devices D4, D5 and D6, showing that the aptamer remain immobilized in the paper after rinsing.

Based on the above studies, we printed devices on paper using a laser printer and then added silver paint contacts (Fig. 1(a)). The purpose of the printed device is to clearly show the location of the Zika solution (or fluids that may contain Zika) on the device. Fig 6(a) shows the $\mathrm{V}_{\text {oc }}$ vs time characteristics response for the device (D1, D2, and D3) where the $V_{\text {oc }}$ dropped almost immediately when the Zika was introduced. The negative $\Delta V_{\text {oc }}$ shift is shown in the respective plots in Fig $6(\mathrm{~b})$. The aptamer stays immobilized in the paper even after rinsing with DI water. Fig 6(c) shows the response of adding Zika, on the three different aptamer coated printed devices (D4, D5, D6). The Voc levels in Fig. 6(c) are almost similar and consistent with that of aptamer-coated device in Fig 6(b).

Although the experiments reported here are straight forward, the actual interactions between different components in the paper matrix is quite involved and the sensor response depends on the history of the paper, temperature, air current on the sensor, and other environmental factors, leading to higher $V_{\text {oc }}$ variations [15] than in micro-fabricated devices. However, the results shown here are in agreement with the overall trend seen with many averaged measurement results (with a standard deviation of $\sim 2 \mathrm{mV}$ ) and theory. Also, the paper devices were manually cut $(\sim 1 \mathrm{~cm} \times 0.2 \mathrm{~cm})$. Using machine controlled automated cutting techniques, variations in device dimensions can be reduced considerably. Uniformity of the electrical contacts was another issue that can be addressed using 3-D printing. Another very important source of error was in making sure that the paper sensor was not saturated with the analytes introduced at each step. When the paper was saturated, the addition of Zika resulted in a thin layer of liquid above the paper sensor and Zika did not incorporate in the paper resulting in erratic sensor output voltages. The sensor was also very sensitive to spurious environmental electric fields generated by us and other objects nearby that could be addressed by proper grounding.

\section{Theoretical Background}


The charge distribution and ionic transport of different charged species in the paper device and the resulting open circuit voltage, can be explained using phase boundary model formed by two different ionic species (aptamer in the background and Zika added on the positive side). The electrochemical potential difference is a sum of the phase boundary potentials [31] between the Zika/aptamer-electrode ( $\mathrm{V}_{\text {oc1 }}$ ), the liquid junction potential [32], [33] between the Zika/aptameraptamer $\left(\mathrm{V}_{\mathrm{oc}}\right)$ and between the aptamer-electrode due to concentration difference of the Zika between the reference aptamer-buffer soaked paper $\left(\mathrm{V}_{\text {ocs }}\right)$, results in the sum-total open circuit voltage or the zero-current potential $\left(\mathrm{V}_{\mathrm{oc}}=\mathrm{V}_{\mathrm{oc}}+\mathrm{V}_{\mathrm{oc} 2}+\mathrm{V}_{\mathrm{oc}}\right)$. Fig 7 shows the schematic of the phase boundary between electrodes and the paper and liquid junction potential at the center. The phase boundary potential between the Zika/aptamer-electrode interface is obtained from the Nernst equation and has a logarithmic dependence with the concentration of Zika sample.

$$
\begin{gathered}
{\left[Z_{Z i k a^{-}}\right] \rightleftharpoons[Z i k a]+e^{-}} \\
V_{o c 1}=V_{0}-\frac{R T}{n_{1} F} \ln \frac{1}{\left[Z i k a^{-}\right]} .
\end{gathered}
$$

where, $V_{0}$ is the constant standard potential $(\mathrm{V}), \mathrm{R}$ is the gas constant, $\mathrm{T}$ is temperature $(\mathrm{K}), \mathrm{F}$ is the faraday constant $(\mathrm{C} / \mathrm{mol})$ and $\mathrm{n}_{1}$ is the number of electron $(\mathrm{mol})$. The phase boundary potential at the aptamer-electrode interface is similar to above expression and is given by, $V_{o c 3}=V_{0}$ $\frac{R T}{n_{3} F} \ln \frac{1}{\left[\text { aptamer }^{-}\right]^{\prime}}$, where the symbols have their usual meaning. The liquid junction potential between Zika/aptamer-aptamer can be written in the form [31, 32],

$$
V_{o c 2}=-\sum_{i} \frac{R T}{F z_{i}} \int_{1}^{m} t_{i} d\left(\ln C_{i}\right)
$$

where, $z_{i}$ is the signed valence of the particular sample species, $C_{i}$ denotes the activity of the ions/sample species and $t_{i}$ is the transference number and signifies the fractional conductance of the $i^{\text {th }}$ ion/sample species. The transference number defined by $t_{i}=\frac{\left|z_{i}\right| u_{i} M_{i}}{\sum_{j}\left|z_{j}\right| u_{j} M_{j}}$, where $u$ is the mobility of the $i^{\text {th }}$ ion and $M$ is the molar concentration, with $j$ ranging over all the ions. An electro neutrality region is assumed to be formed, as the Zika is added on one side. The neutral region is formed by charge separation of the negatively charged Zika and the cations across the junction interface due to their different free energies in the two phases [31]. As can be seen in Fig. 2, the buffer has positive residual charge. On the other hand, the Zika has negative residual charge (Fig. 3). A net electric dipole forms between the positive buffer and nearly zero charge density buffer + Zika regions where the compensating positive charges of the buffer cancels the negative charge of the Zika or Zika/aptamer conjugate. This resulted in different $\Delta \mathrm{V}_{\mathrm{oc}}$ for Zika only and Zika/aptamer conjugate. The Zika/aptamer conjugate provided higher $\mathrm{V}_{\text {oc }}$ change than Zika only. The reason can be attributed to the aptamer configuration change and charge redistribution after binding with Zika virus. Additionally, Zika (40 nm diameter) becomes immobilized in the paper fibers with the aptamers (reason discussed earlier with the AFM studies), hence there is no diffusion of Zika species through the interface, which allows phase boundary model to be implemented in this scenario. The selectivity of Zika is addressed with the use of Zika aptamer on paper discussed earlier.

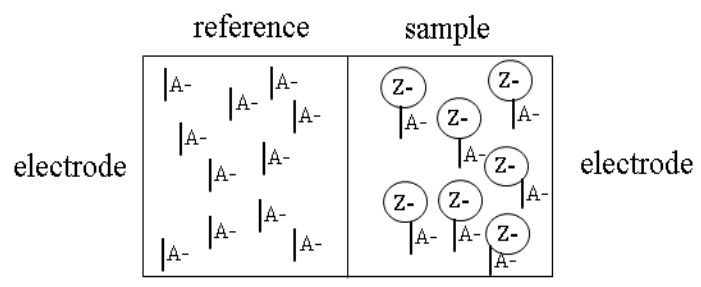

Figure 7. Schematic of the phase-boundary potential model with the electrodes and liquid wherein the potential is given by uneven distribution of the sample ions/particles.

\section{Electronic readout from paper device}


We next explored the possibility of detecting the virus from the paper-based sensor in a form of direct electronic readout through an LCD instead of measuring the open circuit voltage using a voltmeter. The LCD can be configured to display any letter including " $Z$ " once the paper sensor voltage reaches a threshold value generated by the presence of sufficient number of Zika viruses. In order to demonstrate the feasibility of such application, we used an LCD screen from a digital watch and experimentally obtained turn-on voltage for a single display segment to be $\sim 100 \mathrm{mV}$. From the previous results, we observed, Zika when added on one side of the on each of the aptamer- soaked paper device typically produces $\sim 50 \mathrm{mV}$. This open circuit voltage can be increased by adding paper sensors in series. 6 paper sensors can be used in series to generate $\sim 300 \mathrm{mV}$ or more as demonstrated in Fig 8(a). The only drawbacks are that sensors should be fed Zika separately and the external load should not sink much current to cause internal sensor resistor to become important. The paper strip in these sensors connected in series was soaked in aptamer and then Zika was added to one side, showing the letter " $\mathbf{A}$ " as can be seen in Fig $8(\mathrm{~b})$ in the LCD indicating the presence of Zika. The LCD can be replaced by electronic-ink display which requires much low power and hence would eliminate the necessity of using paper device in series.

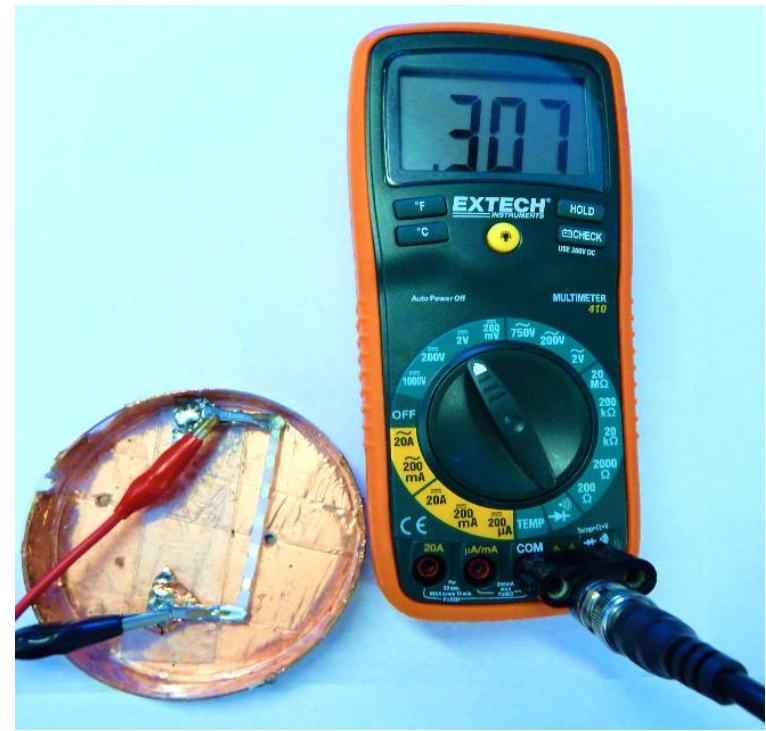

(a)

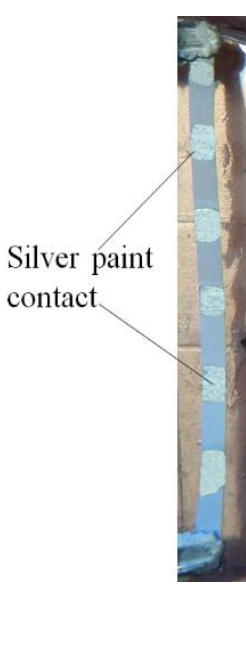

(b)

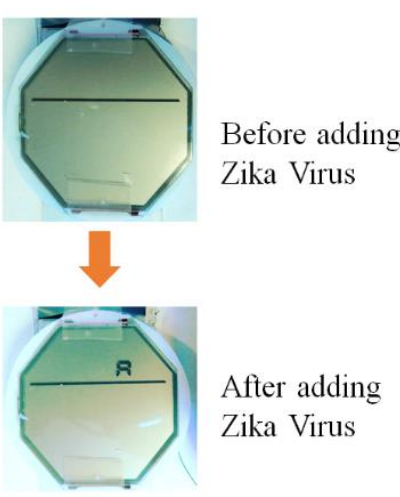

Figure 8. a) Output from the paper sensor measured using multimeter showing voltage level of $\sim 300$ $\mathrm{mV}$ for 6 paper sensors connected in series. b) Output from the paper sensor connected to the LCD readout optioned from digital watch showing the letter " $\mathrm{A}$ " on adding Zika on each side of each of the aptamer-soaked paper devices. The paper strip is connected using clips with 6 devices imprinted on the strip. The grey patches are the silver contact paint regions separating the sensors from each other.

The paper sensors we constructed were very reproducible as long as they were not saturated and grounded properly. Their sensitivity of $2.6 \mathrm{nV} / \mathrm{Zika}$ and their minimum detectable signal (MDS) of $1.2 \times 10^{6} \mathrm{Zika}$ are quite good given their simple structures and ease of operations. Selectivity of this sensor as well as other aptamer-based sensors are limited by the selectivity of their respective aptamers that can be modified to achieve better selectivity if needed. In the case of paper sensor discussed here the selectivity may also be affected by viruses becoming immobilized in the paper even if they are do not bind with the resident aptamer. These other viruses can be removed by mildly washing them away that will not affect the Zika-aptamer complexed appreciably. The real-world application of Zika paper sensors will involve presence of bodily fluids such as urine or sweat or saliva. These biofluids are very complex and proper filtering on the sensor will be used to remove their components that may affect the paper sensor operation adversely.

\section{Conclusion}


This work demonstrates for the first time a paper-based sensor for detecting Zika virus using potentiometry. The sensor had a minimum sensitivity of $2.6 \mathrm{nV} / \mathrm{Zika}$ and a minimum detectable signal of $1.2 \times 10^{6} \mathrm{Zika}$. It can be generalized to detect any other viruses, pathogens even bacteria by simply using appropriate aptamers. The most important and unique aspect of the potentiometric paper based Zika virus is its structural simplicity and ease of use. This study demonstrates the feasibility of detecting whole Zika virus and the use of simple electronic readout. Although the aptamer is known for its high specificity to capture the target pathogen, more study is required with different viruses for its cross-sensitivity and use of different serum like (urine, saliva) for crossreactivity study, in order to use as a reliable field-deployable sensor.

Acknowledgments: This research was supported by NSF (National Science Foundation) Grant under Dr. Leon Esterowitz. The authors also acknowledge contributions of Dr. Jules Magda and Tram Nguyen, Chemical Engineering Department, University of Utah, USA for Aptamer activation and Zika virus handling and processing.

\section{References}

[1] A. Määttänen et al., "A low-cost paper-based inkjet-printed platform for electrochemical analyses," Sensors Actuators, B Chem., vol. 177, pp. 153-162, 2013.

[2] A. C. Siegel, S. T. Phillips, M. D. Dickey, N. Lu, Z. Suo, and G. M. Whitesides, "Foldable printed circuit boards on paper substrates," Adv. Funct. Mater., vol. 20, no. 1, pp. 28-35, Jan. 2010.

[3] D. Tobjörk and R. Österbacka, "Paper electronics," Adv. Mater., vol. 23, no. 17, pp. 19351961, May 2011.

[4] M. Novell, T. Guinovart, P. Blondeau, F. X. Rius, and F. J. Andrade, "A paper-based potentiometric cell for decentralized monitoring of Li levels in whole blood," Lab Chip, vol. 14, no. 7, pp. 1308-1314, Mar. 2014.

[5] J. Cui, G. Lisak, S. Strzalkowska, and J. Bobacka, "Potentiometric sensing utilizing paperbased microfluidic sampling," Analyst, vol. 139, no. 9, pp. 2133-2136, Apr. 2014.

[6] J. Szucs and R. E. Gyurcsányi, “Towards Protein Assays on Paper Platforms with Potentiometric Detection," Electroanalysis, vol. 24, no. 1, pp. 146-152, Jan. 2012.

[7] J. Hu, A. Stein, and P. Bühlmann, “A Disposable Planar Paper-Based Potentiometric IonSensing Platform," Angew. Chemie - Int. Ed., vol. 55, no. 26, pp. 7544-7547, Jun. 2016.

[8] P. Sjöberg et al., "Paper-based potentiometric ion sensors constructed on ink-jet printed gold electrodes," Sensors Actuators, B Chem., vol. 224, pp. 325-332, 2016.

[9] E. W. Nery and L. T. Kubota, "Integrated, paper-based potentiometric electronic tongue for the analysis of beer and wine," Anal. Chim. Acta, vol. 918, pp. 60-68, Apr. 2016.

[10] J. H. Jin, J. H. Kim, S. K. Lee, S. J. Choi, C. W. Park, and N. K. Min, “A fully integrated papermicrofluidic electrochemical device for simultaneous analysis of physiologic blood ions," Sensors (Switzerland), vol. 18, no. 1, 2018.

[11] J. Kofler, S. Nau, and E. J. W. List-Kratochvil, "A paper based, all organic, referenceelectrode-free ion sensing platform," J. Mater. Chem. B, vol. 3, no. 25, pp. 5095-5102, 2015.

[12] J. Ding, N. He, G. Lisak, W. Qin, and J. Bobacka, "Paper-based microfluidic sampling and separation of analytes for potentiometric ion sensing," Sensors Actuators, B Chem., vol. 243, pp. 346-352, 2017.

[13] M. Parrilla, R. Cánovas, and F. J. Andrade, “Paper-based enzymatic electrode with enhanced 
potentiometric response for monitoring glucose in biological fluids," Biosens. Bioelectron., vol. 90, pp. 110-116, 2017.

[14] G. Lisak, J. Cui, and J. Bobacka, "Paper-based microfluidic sampling for potentiometric determination of ions," Sensors Actuators, B Chem., vol. 207, no. PB, pp. 933-939, 2015.

[15] R. Kawahara, P. Sahatiya, S. Badhulika, and S. Uno, "Paper-based potentiometric pH sensor using carbon electrode drawn by pencil," Jpn. J. Appl. Phys., vol. 57, no. 4, p. 04FM08, Apr. 2018.

[16] J. Ping, Y. Wang, K. Fan, W. Tang, J. Wu, and Y. Ying, “High-performance flexible potentiometric sensing devices using free-standing graphene paper," J. Mater. Chem. B, vol. 1, no. 37, pp. 4781-4791, 2013.

[17] A. A. Al-Qahtani, N. Nazir, M. R. Al-Anazi, S. Rubino, and M. N. Al-Ahdal, “Zika virus: a new pandemic threat," J Infect Dev Ctries, vol. 10, no. 3, pp. 201-207, 2016.

[18] C. J. Haug, M. P. Kieny, and B. Murgue, "The zika challenge," New England Journal of Medicine, vol. 374, no. 19. Massachussetts Medical Society, pp. 1801-1803, 12-May-2016.

[19] J. Shin, A. G. Cherstvy, and R. Metzler, "Sensing Viruses by Mechanical Tension of DNA in Responsive Hydrogels," APS, vol. 4, no. 2, 2014.

[20] K. Murray, R. Gorchakov, ... A. C.-E. infectious, and undefined 2017, "Prolonged detection of Zika virus in vaginal secretions and whole blood," ncbi.nlm.nih.gov.

[21] G. Calvet, R. Aguiar, A. Melo, ... S. S.-T. L. infectious, and undefined 2016, “Detection and sequencing of Zika virus from amniotic fluid of fetuses with microcephaly in Brazil: a case study," Elsevier.

[22] O. Faye, O. Faye, A. Dupressoir, ... M. W.-J. of C., and undefined 2008, “One-step RT-PCR for detection of Zika virus," Elsevier.

[23] "Detection of Zika virus in urine," ncbi.nlm.nih.gov.

[24] C. Klungthong et al., "Dengue Virus Detection Using Whole Blood for Reverse Transcriptase PCR and Virus Isolation," J. Clin. Microbiol., vol. 45, no. 8, pp. 2480-2485, 2007.

[25] "Detection of Zika virus in saliva," Elsevier.

[26] K. H. Lee and H. Zeng, "Aptamer-Based ELISA Assay for Highly Specific and Sensitive Detection of Zika NS1 Protein," Anal. Chem., vol. 89, no. 23, pp. 12743-12748, 2017.

[27] R. D. Jenison, S. C. Gill, A. Pardi, and B. Polisky, “High-resolution molecular discrimination by RNA," Science (80-. )., vol. 263, no. 5152, pp. 1425-1429, 1994.

[28] “Zika Envelope Recombinant Antigen I Zika Virus | ProSpec." [Online]. Available: https://www.prospecbio.com/zika_envelope_sf9.

[29] “Base Pair Biotechnologies - Aptamer Discovery Company." [Online]. Available: https://www.basepairbio.com/. [Accessed: 04-Sep-2019].

[30] S. Dolai and M. Tabib-Azar, “433 MHz Lithium Niobate microbalance aptamer-coated whole Zika virus sensor with $370 \mathrm{~Hz} / \mathrm{ng}$ sensitivity," IEEE Sens. J., pp. 1-1, Dec. 2019.

[31] E. Bakker, P. Bühlmann, and E. Pretsch, “The phase-boundary potential model," Talanta, vol. 63, no. 1, pp. 3-20, 2004.

[32] H. H.-C. E. Science and undefined 1970, “The liquid junction potential - the free diffusion junction," Elsevier.

[33] H. W. Harper, "Calculation of liquid junction potentials," J. Phys. Chem., vol. 89, no. 9, pp. 1659-1664, 1985. 


$$
10 \text { of } 10
$$

